\title{
Visual-Functional Mismatch Between Coronary Angiography, Fractional Flow Reserve, and Quantitative Coronary Angiography
}

\author{
Morteza Safi, MD ${ }^{1}$ Vahid Eslami, MD ${ }^{1}$ Mohammad Hasan Namazi, MD ${ }^{1}$ Hossain Vakili, MD ${ }^{1}$ \\ Habib Saadat, MD ${ }^{1}$ Saeid Alipourparsa, MD ${ }^{1}$ Ali Adibi, MD ${ }^{1}$ \\ Mohammad Reza Movahed, MD, PhD, FACP, FACC, FSCAl 2,3
}

${ }^{1}$ Cardiovascular Research Center, Shahid Beheshti University of
Medical Sciences, Tehran, Iran
${ }^{2}$ CareMore Health Care Arizona, Tucson, Arizona
${ }^{3}$ University of Arizona College of Medicine, Tucson, Arizona

Address for correspondence M. Reza Movahed, MD, PhD, FACP, FACC, FSCAI, CareMore Health Care, 7091 East Speedway Boulevard, Tucson, AZ 85710 (e-mail: rmova@aol.com).

Int J Angiol 2016;25:229-234.

\begin{abstract}
Keywords

- angiography

- ischemia

- QCA

- FFR

- coronary imaging

- stenosis

- coronary intervention

Anatomical and functional mismatches are not uncommon in the assessment of coronary lesions. The aim of this study was to identify clinical and lesion-specific factors affecting angiographic, anatomical, and functional mismatch in intermediate coronary lesions. In patients who underwent coronary angiography for clinical reasons, fractional flow reserve (FFR), and quantitative coronary angiography (QCA) analyses for intermediate stenotic lesions were performed simultaneously. Mismatches between the measured values were analyzed. A total of 95 intermediate lesions were assessed simultaneously by visual angiography, FFR, and QCA. The visual-FFR mismatch was found in $40 \%$ of the lesions while reverse visual-FFR mismatch was determined in nearly $14 \%$ of the lesions. Mismatch and reverse mismatch between FFR and QCA parameters were observed in 10 and $23 \%$ of the lesions. FFR value was significant in $32 \%$ of the lesions while visually significant stenosis was shown in $61 \%$ of the lesions. Among the visual-FFR reverse mismatch group, the prevalence of culprit lesions within the left anterior descending (LAD) was significantly higher than other vessels ( $p$ value $<0.02$ ). There were high frequencies of angiographic, QCA, and functional mismatches in analyses of intermediate coronary lesions. LAD lesions showed the highest mismatch. Angiographic or QCA estimation of lesion severity has consistently resulted in inappropriate stenting of functionally nonsignificant lesions or undertreatment of significant lesions based on FFR.
\end{abstract}

Patients with symptomatic ischemia have the most benefit from percutaneous coronary intervention (PCI). An intermediate coronary lesion seen on angiography can be under- or overestimated using visual estimation. Intermediate lesions are defined as a luminal narrowing area between 40 and $70 \%$ diameter stenosis (DS). Assessment of a coronary lesion with intermediate severity continues to be a challenge for the cardiologists. ${ }^{1}$ Unfortunately, noninvasive imaging techniques, as well as coronary angiography are flawed with inaccuracy particularly in obese patients or in patients with multivessel coronary artery disease. This has resulted in inappropriate stenting of functionally nonsignificant lesions, and in some cases, inappropriate deferral of PCI of significant lesions.

Due to coronary arteriography limitation using visual estimation, quantitative coronary angiography (QCA) has been used to improve accuracy. The greatest advantage of published online

December 31, 2015
Copyright @ $₫ 2016$ by Thieme Medical Publishers, Inc., 333 Seventh Avenue, New York, NY 10001, USA. Tel: +1(212) 584-4662.
DOI http://dx.doi.org/ 10.1055/s-0035-1569992. ISSN 1061-1711. 
QCA is its theoretical observer independence minimizing significant potential intraobserver and interobserver variability. In prospective studies, the potential for observer error with visual analysis from a coronary angiogram has been estimated to exceed 35\%. Perhaps the single greatest drawback of QCA is the lack of correlation between the functional predictions of angiographic disease severity and directly measured physiologic variables, as they relate to coronary flow and fractional flow reserve (FFR). ${ }^{2-4}$ Because of the numerous other factors contributing to resistance to blood flow, differences between the anatomic and physiologic data can be expected, most often in the assessment of disease severity, especially in the lesions with intermediate stenosis. To overcome this limitation, physiologic testing is often performed before proceeding with coronary interventions. The functional significance of coronary stenotic lesions is determined not only by the degree of stenosis, but also by viability of myocardial tissue supplied by the lesion, collaterals, and vasomotor tone among others. These physical constraints and resultant flow characteristics contribute to the disparity between the angiographic and corresponding physiologic assessment of disease severity. ${ }^{5}$ An intermediate stenosis, defined as a 40 to $70 \%$ diameter narrowing is encountered in almost $50 \%$ of the patients undergoing coronary arteriography. FFR measurements are consistent with stress testing results and can identify important lesions, thereby assisting in immediate decision-making at the time of cardiac catheterization. The accuracy of FFR for assessment of the functional significance of a coronary stenosis has been well established. ${ }^{6}$ In the DEFER study, nonsignificant stenosis (as indicated by a FFR $>0.75$ ) was randomized to be treated either medically or by stenting. After a follow-up of 5 years, the prognosis of those lesions with FFR $>0.75$ treated medically was excellent with a mortality and myocardial infarction (MI) rate of $<1 \%$ per year and was not improved by stenting. In the FFR-guided arm of the fractional flow reserve versus angiography for multi vessel evaluation (FAME) study, lesions with an FFR $<0.80$ were stented, while PCI was deferred in lesions with FFR $>0.80$. After a follow-up of 2 years, outcome of the deferred lesions was excellent with medical treatment yielding a $<1 \%$ rate of MI or death. ${ }^{7}$ FFR-guided $\mathrm{PCI}$ reduces the need for revascularization for many intermediate lesions. ${ }^{8}$ Moreover, FFR-guided coronary artery bypass grafting (CABG) was associated with a lower number of graft anastomoses and lower rate of on-pump surgery compared with angiography-guided $C A B G$ without a higher event rate during follow-up. ${ }^{9}$

Coronary angiography often underestimates or overestimates lesion's functional severity. ${ }^{3-11}$ A subanalysis of the FAME trial demonstrated that two-thirds of coronary lesions with a DS > 50\% were not ischemia-producing. Conversely, for left main lesions, approximately one-fifth of the lesions with a DS $<50 \%$ were ischemia-producing. Although such a "visual-functional mismatch" is frequently encountered, the mechanism of this phenomenon is poorly understood. ${ }^{10}$ The aim of this study was to identify clinical and lesion-specific factors affecting angiographic, anatomical, and functional mismatch in intermediate coronary lesions.

\section{Methods}

This study was a prospective single-center observational study. This study was approved by the institutional review board. All patients enrolled provided written informed consent. All patients in this study underwent routine coronary angiography due to clinical indications. To evaluate physiologically significant intermediate coronary stenosis (defined between 40 and $70 \%$ by visual estimation), FFR study was performed and QCA was measured. Visual estimation of the lesion severity was compared with FFR and QCA data. Factors affecting their mismatch were analyzed.Exclusion criteria included multiple stenosis (DS $>40 \%$ on visual estimation) within a single target vessel (lesions were considered separate if they were more than three reference vessel diameters apart), bypass graft lesions, left main lesions, side branch lesions, in-stent restenosis, previous $\mathrm{PCI}$ in the target vessel, culprit vessels in the setting of a MI, history of MI in target vessel territory, thrombolysis in MI flow grade $<3$ and angiographic thrombi-containing lesions. Angiographic visual, FFR "mismatch" was defined as angiographic visual DS $>50 \%$ coupled with FFR $>0.80$, whereas "reverse mismatch" was defined as angiographic visual DS $\leq 50 \%$ coupled with $\mathrm{FFR} \leq 0.80$. For QCA-FFR analysis, minimum luminal diameter (MLD) \% $\geq 50 \%$ was considered significant and mismatch and reverse mismatch between their results were also evaluated. Treatment strategies were determined based on the operator's decision.

\section{Fractional Flow Reserve}

"Equalizing" was performed with the guide wire sensor positioned at the guiding catheter tip. The 0.014-in pressure guide wire (Radi, St. Jude Medical, Uppsala, Sweden) was then advanced distal to the stenosis. FFR was measured at the maximal hyperemia induced by intracoronary administration of $200 \mu \mathrm{g}$ of nitroglycerin, and infusion of intra coronary adenosine $(200 \mu \mathrm{g}$ for right coronary artery and $300 \mu \mathrm{g}$ for left anterior descending [LAD] or left circumflex artery). Stenosis was considered significant when FFR was $<0.80$.

\section{Quantitative Coronary Angiography}

Patients initially underwent routine coronary angiography. Angiographic cine images were acquired at 15 frames per second (Axiom Artis II, Siemens, Germany). Two-dimensional (2D)-QCA was performed offline using standard commercial software, Syngo, Siemens. All analyses were performed during the electrocardiographic-gated end-diastolic frame. Angiographic views with the least foreshortening and yielding the best depiction of the stenosis were used. Edge detection correction was performed if required. Minimum luminal area, percentage area stenosis, MLD, percentage DS, and lesion length were measured using 2D-QCA. All measurements were performed twice and averaged by a single experienced cardiologist blinded to the FFR results. Interobserver error was determined by a second cardiologist. 


\section{Results}

In this study, 95 lesions among 72 eligible patients who underwent coronary angiography were assessed. The baseline characteristics of patients are provided in - Table $\mathbf{1}$. The average age was 59.7 years. Overall, 53 (56\%) of patients were males and 42 (44\%) were females. A total of $34 \%$ of patients had a history of diabetes. Acute coronary syndrome comprising non-ST segment elevation myocardial infarction (NSTEMI) and unstable angina was the presenting clinical symptom in $43 \%$ of patients. Visual-FFR mismatch was found in $40 \%$ of the lesions while reverse visual-FFR mismatch was determined in nearly $14 \%$ of the lesions (-Table 2 ). In addition, mismatch and reverse mismatch between FFR and QCA parameters were observed in 10 lesions (10\%) and 22 lesions (23\%), respectively (-Table 3 ). The age of patients in the significant FFR group was higher than the nonsignificant group, but their

Table 1 Baseline characteristics of patients

\begin{tabular}{|l|l|}
\hline Patients characteristics & $N(\%)$ \\
\hline Age (y) (mean \pm SD) & $59.7 \pm 8.9$ (range, 41-86) \\
\hline Sex & $53(56 \%)$ \\
\hline Male (\%) & $42(44 \%)$ \\
\hline Female (\%) & $36(37.9)$ \\
\hline Risk factors & $43(45.3)$ \\
\hline Diabetes mellitus (\%) & $40(42.1)$ \\
\hline HTN (\%) & $21(22.1)$ \\
\hline Dyslipidemia (\%) & \\
\hline Smoking (\%) & $54(56.8)$ \\
\hline Symptom on admission & $40(42.10)$ \\
\hline Stable angina & $1(1.1)$ \\
\hline Unstable angina & $58(61.1)$ \\
\hline NSTEMI & $17(17.9)$ \\
\hline Vessel & $20(22)$ \\
\hline LAD & \\
\hline LCX & \\
\hline RCA & \\
\hline
\end{tabular}

Abbreviations: HTN, hypertension; LAD, left anterior descending; LCX, left circumflex artery; NSTEMI, non-ST segment elevation myocardial infarction; RCA, right coronary artery; SD, standard deviation. difference did not show any statistical significance ( - Tables 2 and 3). The differences in coronary risk factor prevalence and ejection fractions of patients in subgroup analyses were not significant. LAD was the most common culprit vessel evaluated in this study (58 cases, 61\%). Distribution of culprit stenosis is shown in -Table 4. FFR value was significant in 31 lesions (32\%), while visually significant stenosis was shown in 58 lesions (61\%). Moreover, anatomically significant stenosis based on QCA parameters (defined as MLD\% $\geq 50$ ) was only found in $22 \%$ of the lesions. Analysis of culprit vessels showed that in visual-FFR mismatch and reverse mismatch groups, LAD was the most commonly involved vessel with 57.9 and $71.4 \%$, respectively. Prevalence in QCA-FFR mismatch and reverse mismatch groups was 40 and $66 \%$, respectively. Among the visual-FFR reverse mismatch group, the prevalence of culprit lesions within the LAD was significantly higher than other vessels ( $p$ value $<0.02$ ). In the QCA-FFR mismatch group, the LAD was significantly more involved than the other vessels ( $p$ value $<0.04$ ). Acute coronary syndrome was a significantly more common clinical presentation in patients with visual-FFR reverse mismatch group ( $p$ value $<0.004$ ), while there was no significant difference in the clinical presentation of the visual-FFR mismatch group ( $p$ value $=0.44)$. The correlations between FFR data and QCA parameters showed positive modest correlations between MLD and reference diameter with FFR ( $p$ value $<0.01$, -Figs. 1 and 2 ).

\section{Discussion}

Earlier studies showed that FFR $<0.75$ is an accurate predictor of ischemia while more recent studies have used FFR $\leq 0.80$ as a cutoff to guide revascularization; thus, we used 0.80 as a cut-off value for significant FFR in our analysis. ${ }^{11,12}$ In this prospective study, our data demonstrated that visual-functional mismatch between coronary angiography and FFR was frequently encountered (as high as 53\%) and mismatch between QCA parameters and FFR was also as high as $34 \%$ which is in agreement with results of previous studies by Park et al that showed $40 \%$ mismatch. ${ }^{13}$ The DS by visual estimation was significantly greater compared with DS calculated by QCA analysis which was consistent with findings of multiple trials. ${ }^{13}$ The physiological effect of stenosis depends on clinical and local factors. FFR reflects the sum of all these factors and the reliability of FFR results for decision making has been

Table 2 Vessel and lesion distribution

\begin{tabular}{|c|c|c|c|c|c|c|}
\hline & \multicolumn{4}{|c|}{ Stenosis site } & \multirow[t]{2}{*}{ Total } \\
\hline & & Origin & Proximal & Mid & Distal & \\
\hline \multirow[t]{3}{*}{ Vessel } & LAD & $\begin{array}{l}10 \\
(17.2 \%)\end{array}$ & $\begin{array}{l}28 \\
(48.3 \%)\end{array}$ & $\begin{array}{l}20 \\
(34.5 \%)\end{array}$ & 0 & 58 \\
\hline & LCX & $2(11.8 \%)$ & $4(23.5 \%)$ & 11 (64.7\%) & 0 & 17 \\
\hline & RCA & 0 & $7(35 \%)$ & $9(45 \%)$ & $4(20 \%)$ & 20 \\
\hline \multicolumn{2}{|l|}{ Total } & 12 & 39 & 40 & 4 & 95 \\
\hline
\end{tabular}

Abbreviations: LAD, left anterior descending; LCX, left circumflex artery; RCA, right coronary artery. 
Table 3 Demographic, angiographic, and visual stenosis values parameters

\begin{tabular}{|c|c|c|c|c|c|c|}
\hline & \multicolumn{3}{|c|}{$\begin{array}{l}\text { Visual stenosis values } \leq 50 \% \\
(n=37)\end{array}$} & \multicolumn{3}{|c|}{$\begin{array}{l}\text { Visual stenosis values }>50 \% \\
(n=58)\end{array}$} \\
\hline & \multicolumn{3}{|l|}{ FFR value } & \multicolumn{3}{|l|}{ FFR value } \\
\hline & $\begin{array}{l}\text { Significant- } \\
\text { stenosis }^{13} \\
\text { (Reverse mis- } \\
\text { match group) }\end{array}$ & $\begin{array}{l}\text { Nonsignificant- } \\
\text { stenosis (24) }\end{array}$ & $p$ Value & $\begin{array}{l}\text { Significant- } \\
\text { stenosis (20) }\end{array}$ & $\begin{array}{l}\text { Nonsignificant- } \\
\text { stenosis (38) } \\
\text { (Mismatch group) }\end{array}$ & $p$ Value \\
\hline Age & $61.92 \pm 9.88$ & $60.15 \pm 8.77$ & 0.8 & $64.17 \pm 7.12$ & $56.8 \pm 10.65$ & 0.1 \\
\hline $\operatorname{Sex}(M / F)$ & $3 / 4(42.9 / 57.1)$ & $13 / 11(54.2 / 45.8)$ & 0.62 & $10 / 10(50 / 50)$ & $26 / 12(68.4 / 31.6)$ & 0.16 \\
\hline DM (\%) & $5(38.5)$ & $12(50)$ & 0.52 & $4(20)$ & $15(39.5)$ & 0.12 \\
\hline HTN (\%) & $6(46.2)$ & $15(62.5)$ & 0.33 & $8(40)$ & $14(36.8)$ & 0.81 \\
\hline Dyslipidemia (\%) & $5(71.4)$ & $7(53.8)$ & 0.65 & $7(35)$ & $15(39.5)$ & 0.72 \\
\hline Smoking (\%) & $1(7.7)$ & $6(25)$ & 0.38 & $4(20)$ & $10(26.3)$ & 0.59 \\
\hline MLD (\%) & $40.30 \pm 14.39$ & $39.54 \pm 10.27$ & 0.85 & $47.85 \pm 13.84$ & $42.86 \pm 11.56$ & 0.51 \\
\hline Symptom & & & $0.004^{\mathrm{a}}$ & & & 0.44 \\
\hline 1 stable angina & $4(30.8)$ & $19(79.2)$ & & $9(45)$ & $22(57.9)$ & \\
\hline 2 unstable angina & $9(69.2)$ & $5(20.8)$ & & $11(55)$ & $15(39.5)$ & \\
\hline 3 NSTEMI & $0(0)$ & $0(0)$ & & $0(0)$ & $1(2.6)$ & \\
\hline $\mathrm{EF}$ & $47.69 \pm 10.12$ & $45.82 \pm 10.39$ & 0.6 & $49 \pm 10.46$ & $48.42 \pm 6.74$ & 0.4 \\
\hline LAD & $11(84.6)$ & $11(45.8)$ & $0.02^{a}$ & $14(70)$ & 22 (57.9) & 0.52 \\
\hline LCX & $2(15.4)$ & $3(12.5)$ & & $4(20)$ & $8(21.1)$ & \\
\hline RCA & $0(0)$ & $10(41.7)$ & & $2(10)$ & $8(21.1)$ & \\
\hline Original & $2(15.4)$ & $6(25)$ & 0.59 & $2(10)$ & $2(5.3)$ & 0.41 \\
\hline Proximal & $7(53.8)$ & $9(37.5)$ & & $10(50)$ & $13(34.2)$ & \\
\hline Mid & $4(30.8)$ & $7(29.2)$ & & $7(35)$ & 22 (57.9) & \\
\hline Distal & $0(0)$ & $2(8.3)$ & & $1(5)$ & $1(2.6)$ & \\
\hline $\mathrm{FH}$ & $1(7.7)$ & $4(16.7)$ & 0.63 & $4(20)$ & $4(10.5)$ & 0.32 \\
\hline
\end{tabular}

Abbreviations: DM, diabetes mellitus; EF, ejection fraction; F, female; HTN, hypertension; LAD, left anterior descending; LCX, left circumflex artery; M, male; MLD, minimum luminal diameter; NSTEMI, non-ST segment elevation myocardial infarction; RCA, right coronary artery.

${ }^{a} p$ Value $<0.05$ significant.

documented in previous studies. ${ }^{14}$ Our study showed that in the visual-FFR reverse mismatch subgroup, the LAD was the most common culprit vessel which was similar to findings of other studies. ${ }^{13}$ This finding suggests that underestimation of stenosis by visual assessment in the LAD may be due to the larger perfusion area supplied by the LAD compared with other coronary vessels which directly affects the physiological significance of the stenosis expressed in FFR results.

In the visual reverse mismatch group, like other studies, our results also showed that acute coronary syndrome (NSTEMI, unstable angina [UA]) was more common than stable angina ( $p$ value $<0.04)$. This finding maybe related to the shape of plaques in ACS patients that directly influence FFR and make its results significant compared with a similar degree of stenosis in stable plaques. This study showed that in the visual-FFR mismatch group, the LAD was the most common culprit vessel, which was consistent with other researchers. FFR-guided coronary intervention was shown to be superior to 2D-QCA driven coronary intervention in preventing MI, revascularization or death. ${ }^{12}$ Our Study showed that correlation of 2D-QCA parameters with FFR results were weakly positive in this regard and our data were similar to previous reports. ${ }^{15-18}$ This weak correlation could have been related to limitations of 2D-QCA, because it only allows characterization of lesion severity in a single plane while physiologic effects of stenosis represented by FFR were not only related to degree of stenosis but also to features such as myocardial viability and presence of collaterals.

\section{Conclusion}

Our study revealed that relying only on angiographic or noninvasive evaluation or QCA has resulted in inappropriate stenting of functionally nonsignificant lesions and, in some cases, inappropriate deferral of PCI of significant lesions. Mismatch was largest in the LAD lesions suggesting that evaluating intermittent lesions in the LAD territory using visual estimation or QCA alone is inaccurate and FFR should be considered for better assessment. 
Table 4 Demographic, angiographic, and quantitative coronary angiography parameters in patients

\begin{tabular}{|c|c|c|c|c|c|c|}
\hline & \multicolumn{3}{|l|}{$\begin{array}{l}\mathrm{QCA} \leq 50 \% \\
(n=74)\end{array}$} & \multicolumn{3}{|l|}{$\begin{array}{l}\text { QCA }>50 \% \\
(n=21)\end{array}$} \\
\hline & \multicolumn{3}{|l|}{ FFR Value } & \multicolumn{3}{|l|}{ FFR Value } \\
\hline & $\begin{array}{l}\text { Significant- } \\
\text { stenosis (22) } \\
\text { (Reverse } \\
\text { mismatch) }\end{array}$ & $\begin{array}{l}\text { Nonsignificant- } \\
\text { stenosis (52) }\end{array}$ & $p$ Value & $\begin{array}{l}\text { Significant- } \\
\text { stenosis }^{11}\end{array}$ & $\begin{array}{l}\text { Nonsignificant- } \\
\text { stenosis } 10 \\
\text { (Mismatch) }\end{array}$ & $p$ Value \\
\hline Age & $58.48 \pm 8.45$ & $59.67 \pm 8.59$ & 0.54 & $65.72 \pm 8.1$ & $56.8 \pm 10.65$ & $0.04^{a}$ \\
\hline $\operatorname{Sex}(M / F)$ & $9 / 13(40.9 / 59.9)$ & $32 / 20(61.5 / 38.5)$ & 0.16 & $4 / 4(50 / 50)$ & $7 / 3(70 / 30)$ & 0.6 \\
\hline DM (\%) & $8(36.4)$ & $20(38.5)$ & 0.86 & $1(9.1)$ & $7(70)$ & 0.004 \\
\hline HTN (\%) & $10(45.5)$ & $24(46.2)$ & 0.94 & $4(36.4)$ & $5(50)$ & 0.52 \\
\hline Dyslipidemia (\%) & $10(45.5)$ & $21(40.4)$ & 0.8 & $3(36.4)$ & $5(50)$ & 0.52 \\
\hline Smoking (\%) & $4(18.2)$ & $11(21.2)$ & 0.7 & $1(9.1)$ & $5(50)$ & 0.03 \\
\hline Symptom & & & 0.09 & & & 0.03 \\
\hline 1 stable angina & $8(36.4)$ & $32(61.5)$ & & $5(45.5)$ & $9(90)$ & \\
\hline 2 unstable angina & $32(61.5)$ & $19(36.5)$ & & $6(54.5)$ & $1(10)$ & \\
\hline 3 NSTEMI & $0(0)$ & $1(1.9)$ & & & & \\
\hline MLD \% & $38.81 \pm 9.1$ & $38.32 \pm 8.73$ & 0.53 & $61 \pm 7.52$ & $58.5 \pm 5.11$ & 0.3 \\
\hline $\mathrm{EF}$ & $50.41 \pm 9.87$ & $46.82 \pm 8.51$ & 0.28 & $46.81 \pm 13.65$ & $50.50 \pm 7.24$ & 0.45 \\
\hline LAD & $17(77.3)$ & $29(55.8)$ & 0.04 & $8(72.7)$ & $4(40)$ & 0.28 \\
\hline LCX & $4(18.2)$ & $8(15.4)$ & & $2(18.2)$ & $3(30)$ & \\
\hline RCA & $1(4.5)$ & $15(28.8)$ & & $1(9.1)$ & $3(30)$ & \\
\hline Original & $3(13.6)$ & $8(15.4)$ & 0.34 & $1(9.1)$ & $0(0)$ & 0.67 \\
\hline Proximal & $12(54.5)$ & $17(32.7)$ & & $5(45.5)$ & $5(50)$ & \\
\hline Mid & $6(27.3)$ & $24(46.2)$ & & $5(45.5)$ & $5(50)$ & \\
\hline Distal & $1(4.5)$ & $3(5.8)$ & & $0(0)$ & $0(0)$ & \\
\hline
\end{tabular}

${ }^{a} p$ Value $<0.05$ significant.

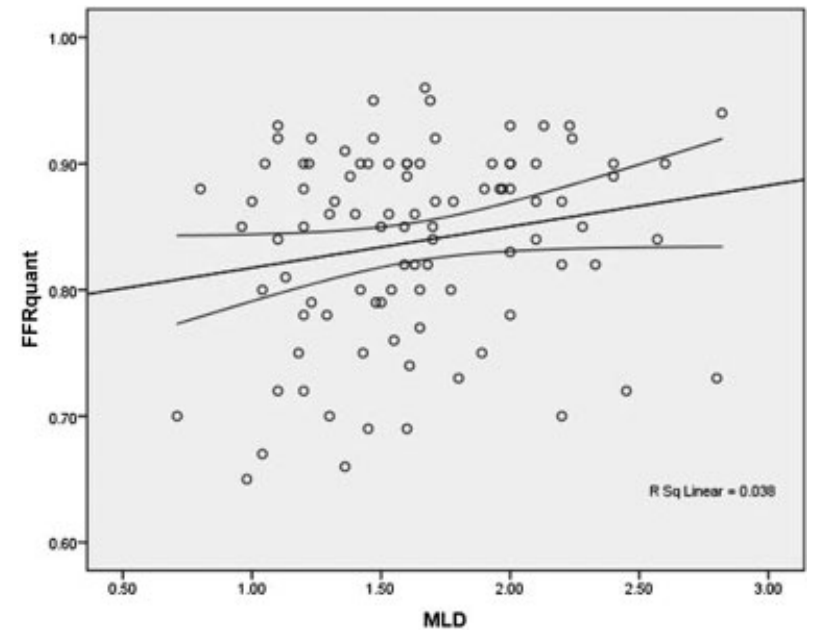

Fig. 1 Correlation between FFR and MLD. FFR, fractional flow reserve; MLD; minimum luminal diameter.

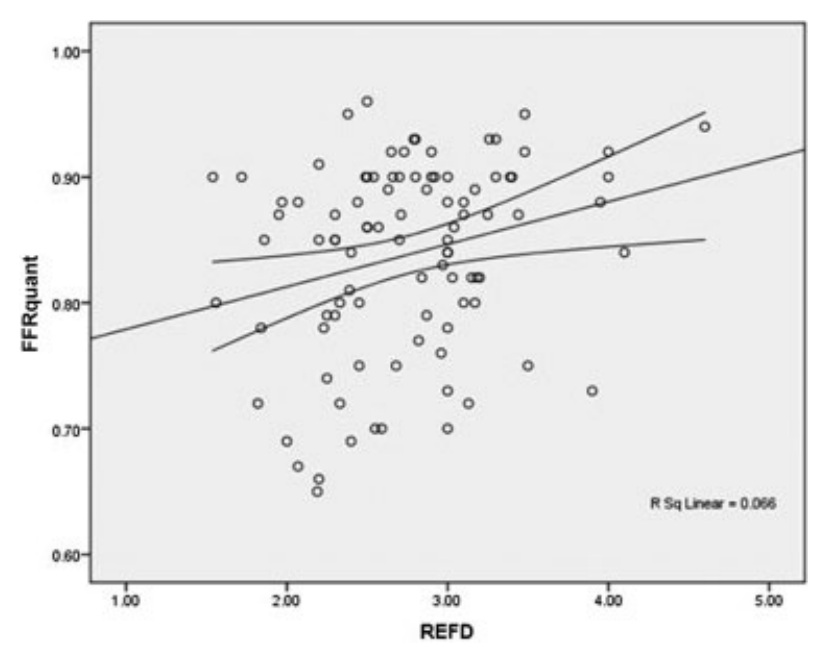

Fig. 2 Correlation between FFR and REFD. FFR, fractional flow reserve; REFD, reference diameter. 


\section{Limitation}

The number of patients might not be large enough limiting our results. We confined our study to patients without left main disease. Furthermore, we excluded patients with history of MI and serial stenosis, which complicated FFR measurements limiting our data to simple lesions.

\section{References}

1 Tobis J, Azarbal B, Slavin L. Assessment of intermediate severity coronary lesions in the catheterization laboratory. 2007;49(8): 839-848

2 Zijlstra F, van Ommeren J, Reiber JH, Serruys PW. Does the quantitative assessment of coronary artery dimensions predict the physiologic significance of a coronary stenosis? Circulation 1987;75(6):1154-1161

3 Kirkeeide RL, Gould KL, Parsel L. Assessment of coronary stenoses by myocardial perfusion imaging during pharmacologic coronary vasodilation. VII. Validation of coronary flow reserve as a single integrated functional measure of stenosis severity reflecting all its geometric dimensions. J Am Coll Cardiol 1986;7(1):103-113

4 Zijlstra F, Fioretti P, Reiber JH, Serruys PW. Which cineangiographically assessed anatomic variable correlates best with functional measurements of stenosis severity? A comparison of quantitative analysis of the coronary cineangiogram with measured coronary flow reserve and exercise/redistribution thallium201 scintigraphy. J Am Coll Cardiol 1988;12(3):686-691

5 Gould KL. Percent coronary stenosis: battered gold standard, pernicious relic or clinical practicality? J Am Coll Cardiol 1988; 11(4):886-888

6 Li J, Elrashidi MY, Flammer AJ, et al. Long-term outcomes of fractional flow reserve-guided vs. angiography-guided percutaneous coronary intervention in contemporary practice. Eur Heart J 2013;34(18):1375-1383

7 Pijls NH, Tanaka N, Fearon WF. Functional assessment of coronary stenoses: can we live without it? Eur Heart J 2013;34(18): $1335-1344$
8 Nam CW, Yoon HJ, Cho YK, et al. Outcomes of percutaneous coronary intervention in intermediate coronary artery disease: fractional flow reserve-guided versus intravascular ultrasoundguided.. J Am Coll Cardiol 2010;3(8):812-817

9 Toth G, De Bruyne B, Casselman F, et al. Fractional flow reserveguided versus angiography-guided coronary artery bypass graft surgery. Circulation 2013;128(13):1405-1411

10 Melikian N, De Bondt P, Tonino P, et al. Fractional flow reserve and myocardial perfusion imaging in patients with angiographic multivessel coronary artery disease. JACC Cardiovasc Interv 2010;3(3): 307-314

11 Hamilos M, Muller O, Cuisset T, et al. Long-term clinical outcome after fractional flow reserve-guided treatment in patients with angiographically equivocal left main coronary artery stenosis. Circulation 2009;120(15):1505-1512

12 Tonino PA, De Bruyne B, Pijls NH, et al; FAME Study Investigators. Fractional flow reserve versus angiography for guiding percutaneous coronary intervention. N Engl J Med 2009;360(3):213-224

13 Park SJ, Kang SJ, Ahn JM, et al. Visual-functional mismatch between coronary angiography and fractional flow reserve. JACC Cardiovasc Interv 2012;5(10):1029-1036

14 Tonino PA, Fearon WF, De Bruyne B, et al. Angiographic versus functional severity of coronary artery stenoses in the FAME study fractional flow reserve versus angiography in multivessel evaluation. J Am Coll Cardiol 2010;55(25):2816-2821

15 Bartúnek J, Sys SU, Heyndrickx GR, Pijls NH, De Bruyne B. Quantitative coronary angiography in predicting functional significance of stenoses in an unselected patient cohort. J Am Coll Cardiol 1995; 26(2):328-334

16 Fischer JJ, Samady H, McPherson JA, et al. Comparison between visual assessment and quantitative angiography versus fractional flow reserve for native coronary narrowings of moderate severity. Am J Cardiol 2002;90(3):210-215

17 Yong AS, Ng AC, Brieger D, Lowe HC, Ng MK, Kritharides L. Threedimensional and two-dimensional quantitative coronary angiography, and their prediction of reduced fractional flow reserve. Eur Heart J 2011;32(3):345-353

18 Topol EJ, Nissen SE. Our preoccupation with coronary luminology. The dissociation between clinical and angiographic findings in ischemic heart disease. Circulation 1995;92(8):2333-2342 